\title{
New DTR line selection method in a power system comprising DTR, ESS and RES for increasing RES integration and minimizing load shedding
}

\author{
Ali Shalchi ${ }^{1}$, Maysam Abbasi ${ }^{2}$, Ehsan Abbasi $^{3}$, Behrouz Tousi ${ }^{1 *}$, Gevork B. Gharehpetian ${ }^{4}$ \\ ${ }^{1}$ Department of Electrical Engineering, Urmia University, Urmia, Iran \\ ${ }^{2}$ School of Electrical and Data Engineering, University of Technology Sydney, Sydney, Australia \\ ${ }^{3}$ Department of Electrical Engineering, University of Tabriz, Tabriz, Iran \\ ${ }^{4}$ Department of Electrical Engineering, Amirkabir University of Technology, Tehran, Iran \\ Corresponding author email: $\underline{\text { b.tousi@urmia.ac.ir }}$
}

\begin{abstract}
In this paper, new technologies such as dynamic thermal rating (DTR) technology and energy storage system (ESS) are simultaneously used to optimize the integration of renewable energy sources (RESs) and minimize the load shedding. For achieving the mentioned aims, a new method is proposed to select the candidate lines for the implementation of DTR technologies. The DTR technology is responsible for increasing lines limited capacity. Moreover, optimally placed and sized ESSs save RESs generated power in non-peak-hours and inject it to the network in peak-hours. For validating the performance of the proposed solution, comprehensive simulations are performed in several stages on IEEE RTS-24 and 30 bus test systems networks. To meet the increased power demand, RESs (wind and solar) are optimally allocated by using the Genetic algorithm (GA) in the test systems. Then, ESS devices are optimally sized and placed by using GA. Finally, candidate lines are selected based on the proposed method and DTR devices are added to the systems. Comprehensive comparisons are presented for comparing the previously presented solutions and the proposed one. It is proved that using DTR technology and ESSs along with the proposed line selection method is the superior solution for system operators.
\end{abstract}

Keywords: Renewable Energy Source, Energy Storage System, Curtailment, Dynamic Thermal Rating, Line Selection Methodology, Optimal Integration

\begin{tabular}{|c|c|c|c|}
\hline \multicolumn{4}{|c|}{ Nomenclature } \\
\hline$B_{j k}$ & $\begin{array}{l}\text { Line susceptance difference between buses } \mathrm{j} \text { and } \mathrm{k} \\
\text { (non-reference buses) }\end{array}$ & $C_{g}^{p}()$. & Active power generation cost $[\$ / \mathrm{h}]$ \\
\hline$C_{g}^{R}()$. & Reserve procurement cost $[\$ / \mathrm{h}]$ & $C_{g}^{s u}()$. & Generating unit start-up cost $[\$ / \mathrm{h}]$ \\
\hline$C_{g}^{s d}()$. & Generating unit shut-down cost $[\$ / \mathrm{h}]$ & $D T_{g}$ & Minimum down time of unit $g$ \\
\hline$P_{w, t}^{\operatorname{Max}}$ & Maximum generation of wind plant & $p_{g, \min }^{G}$ & Minimum power output of unit $g$ \\
\hline$p_{g, \max }^{G}$ & Maximum power output of unit $g$ & $p_{j, t}^{D}$ & Active power demand at bus $j$ in period $t$ \\
\hline$P_{P V, t}^{M a x}$ & Maximum generation of solar plant & $S D_{g}$ & Shutdown ramp limit of unit $g$ \\
\hline$T$ & Number of periods of the time span & $U T_{g}$ & Minimumup time of unit $g$ \\
\hline$V O L L$ & Large number & & \\
\hline$\beta_{t}$ & Charge of ESSs in period $t$ & $\gamma_{t}$ & Decharge of ESSs in period $t$ \\
\hline$\theta_{j k}$ & $\begin{array}{l}\text { Voltage angles difference between buses } \mathrm{j} \text { and } \mathrm{k} \\
\text { (non-reference buses) }\end{array}$ & $L S_{t}$ & Load shedding in period $t$ \\
\hline$L_{g}$ & $\begin{array}{l}\text { Number of initial periods during which unit } g \text { must be } \\
\text { offline }\end{array}$ & $G_{g}$ & Number of initial periods during which unit $j$ must be online \\
\hline$p_{g, t}^{\max }$ & Maximum available power output of unit $g$ in period $t$ & $p_{g, t}^{G}$ & Power output of unit $g$ in period $t$ \\
\hline$p_{j, k}^{l}$ & Line active power flow between bus $j$ and $k$ & $P_{j k, \max }^{D T R_{l}}$ & Line power limit in DTR state \\
\hline$P_{j k, \max }^{S T R_{l}}$ & Line power limit in STR state & $P_{g, t}^{u p}$ & Ramp up of generating unit $g$ in period $t$ \\
\hline$P_{g, t}^{d n}$ & Ramp down of generating unit $g$ in period $t$ & $p_{P V, t}$ & Power output of solar plant \\
\hline$p_{w, t}$ & Power output of wind plant & $R D_{g}$ & Ramp-down limit of unit $g$ \\
\hline$R U_{g}$ & Ramp-up limit of unit $g$ & $S_{g, 0}$ & $\begin{array}{l}\text { Number of periods that unit } g \text { has been offline prior to the } \\
\text { first period of the time span }\end{array}$ \\
\hline$S U_{g}$ & Startup ramp limit of unit $g$ & $U_{g, 0}^{G}$ & $\begin{array}{l}\text { Number of periods that unit } g \text { has been online prior to the } \\
\text { first period of the time span (end of period } 0 \text { ) }\end{array}$ \\
\hline$U_{\mathrm{g}, t}^{G}$ & Generating unit on/off binary variable & & \\
\hline
\end{tabular}




\section{Introduction}

Due to problems like global warming, increasing carbon emissions, augmenting high-quality power demand and depletion of fossil fuels, countries are obliged to increase the share of renewable energy resources (RESs) in electricity networks [1-2]. However, using RESs in a wide range can cause some serious challenges in power systems. One of these challenges is the dissimilarity of RESs generation and load curves. For instance, solar power plants maximum generation occurs at noon, while the load peak can be even during the night. This can cause two problems. First, the operator will be forced to curtail the RES production, since the consumers may be completely supplied by thermal generation units which cannot ramp down any further or quickly enough. Besides, this mismatch can result in load shedding during peak hours. According to the National Renewable Energy Laboratory (NREL) and Wind Technologies Market reports, transmission constraints and generator inflexibility have been the most common reasons for RESs curtailment [3. 4]. Another challenge caused by high penetration of RESs in power systems is that these sources are generally placed in remote areas leading to the need for having enough capacity in the transmission lines to transfer their generated power to the load centers [5]. However, nowadays, transmission lines operate close to their maximum limits due to constantly growing electricity demand and also the investment lack and limiting environmental regulations for implementing new lines [6]. Thus, high penetration of RESs can increase the transmission lines congestions risk which in turn can lead to RESs curtailments and load shedding. Therefore, transmission lines congestions and RESs intermittent nature can be considered as the most important problems for RESs high penetration in power systems [7].

Nowadays, there are several suitable solutions such as dynamic thermal rating (DTR) technologies to improve the transmission line ampacity without investing in an additional transmission network. But, most of the transmission lines are rated by the owner/operator based on continuous or static thermal rating (STR) [8]. An overhead line STR is determined by using the worst set of ambient weather conditions expected during a particular season. Thus, STR can limit the lines' transfer capacity even if the real ambient conditions are better than the worst set. To overcome this problem, dynamic thermal rating (DTR) has been introduced, which uses realtime meteorological information to estimate transmission line ratings [9]. Thus, by using DTR, it is possible to use the maximum capacity of transmission lines which can play a big role in increasing the RESs penetration into the power systems. Many investigations have been conducted on the impact of DTR on power networks. Among them, many studies are focusing on the DTR impact on the penetration of RESs, especially wind turbines/farms [10-16]. In [10], it has been shown that DTR consideration has positive effects on the penetration level of wind turbines. The presented results of this paper have demonstrated that implementing DTR technology increases network reliability and results in higher wind energy penetration. In [11], a comprehensive review has been presented about the application of various DTR systems for improving wind energy penetration into the grid. Additionally, there is another group of researches focusing on DTR effects on transmission line congestion and constraints. In [12], it has been shown that using DTR can reduce the curtailments of RESs which is an important economic saving for the system and also a remarkable environmental benefit. In [13], it has been revealed that DTR implementation can help to reduce the congestion costs and load shedding risk. Moreover, a flexible load shedding scheme based on real-time DTR has been introduced in this paper. In [14], the authors proved that DTR has an impressive effect on increasing wind farm penetration. So far, a thorough study has not been conducted about DTR impacts on RESs integration and load shedding.

As already mentioned, energy storage systems (ESS) can be generally used for solving the intermittent behavior of RESs [16-20]. The ESS devices store the surplus produced energy of RESs in off-peak periods and can inject energy when it is needed [16]. A storage modeling has been presented in [17], which allows systematic study of the integration procedure of RES in a power system depending on the roundtrip efficiency and the size of the storage. In [18], it has been suggested that economic deployment of storage, used for the provision of peak capacity, could provide a substantial resource to reduce variable generation curtailment and increase variable generation penetration. In [19], safe integration of renewable along energy with energy storage devices has been discussed to have a reliable and efficient sustainable energy system. In [20], the DTR has been used to reduce the system costs and wind energy curtailment, and the ESS has been used to optimize the system costs but not for RES curtailment. The results indicate that DTR has a positive effect on reducing system costs and ESS curtailment and that using ESS can reduce system costs.

Obviously, the implementation of the DTR equipment on all the transmission lines may not be economic. So one concern in implementing DTR is the identification of suitable transmission lines. Up to now, several methods have been introduced to select candidate lines for DTR installation [2123]. In [21, 22], line selection is based on historical-simulated weather data so the lines with a critical span have been selected as DTR lines. In [23], an approach has been presented to select the best lines, which could have the highest impact on fuel cost. So far, a DTR line selection method has not been introduced to simultaneously consider the power generation costs, load shedding, and RESs integration.

In this paper, the simultaneous application of ESS and DTR technologies is proposed to optimize the integration of RESs and minimize the load shedding. Moreover, a new method based on the generation costs and load shedding indices is proposed for optimal placement of DTR on the transmission lines. To show the advantages of the proposed method, simulations are presented in MATLAB software environment for IEEE RTS-24 and IEEE 30 bus test systems. The paper is summarised the following steps: 
I. Formulating the main problem

II. Optimal allocation and sizing of RESs including both wind farm and a solar park.

III. Optimal placement and sizing of ESSs.

IV. Implementing DTRs on the candidate lines selected by the proposed methodology.

V. Obtaining the final results and comparing the proposed method with others.

It should be mentioned that no studies have been previously performed to investigate the DTR impact on the penetration of solar plants. Second, for the first time, the DTR impact on generation costs, RESs integration and Load shedding is thoroughly studied. Third, the simultaneous application of ESS and DTR technologies is introduced for achieving RES optimal integration and load shedding. Also, a new DTR line selection method is developed considering the load shedding, generation costs, and RES curtailments. Generally, the main contributions of this paper can be listed as:

- Proposing a new DTR line selection method

- Optimal and simultaneous application of three important technologies, i.e. DTR, ESS and RES, in the power system

- Optimal allocation of the mentioned technologies in the power system

For evaluating the proposed method performance, the security-constrained unit commitment problem (SCUC) is used considering DC load flow constraints and ESSs, RESs, and DTR equations. According to the results, the proposed method shows the best performance in comparison to the previously introduced methods.

This paper is organized as follows. In Section 2, the problem formulation is presented which includes DTR modeling, ESS model, wind farm model, solar power plant model, security-constrained unit commitment with DC load flow and DTR, RES and ESS allocating method, proposed DTR line selection method and evaluation criteria. Then, in Section 3, Case study is given. After that, simulation results and comparisons are presented in Section 4. Finally, a conclusion is given in Section 5.

\section{Problem Formulation}

In this section, the problem formulation along with explanations and models are presented.

\subsection{Dynamic Thermal Rating Model}

Under given weather conditions, a conductor thermal rating can be obtained using the heat balance equation of the conductor [25], which is expressed at the following equation:

$$
q_{c}\left(T_{c}, T_{a}, V_{m}, j\right)+q_{r}\left(T_{c}, T_{a}\right)=q_{s}+I^{2} \times R\left(T_{c}\right)
$$

where, $q_{s}$ and $I x^{2} \times R\left(T_{c}\right)$ are the heat gain due to solar radiation and Joule heating due to current flow in the conductor. Also,
$R$ is a function of conductor temperature; and also $q_{c}$ and $q_{r}$ are the heat loss due to convection and longwave radiation, respectively. In [24], all four heat terms $\left(q_{r}, q_{c}, q_{s}\right.$, and $I x^{2} \times R$ $\left(T_{c}\right)$ ) have been calculated in detail. By rewriting (1), the maximum allowable current rating of the conductor can be determined as follows:

$I=\sqrt{\frac{q_{c}+q_{r}-q_{s}}{R\left(T_{c}\right)}}$

According to the above equation, the conductor temperature may change from one span to another one since wind speed and its direction vary along a transmission line. This means that the allowable line thermal capacity could vary in different spans. Thus, the line maximum capacity should be estimated at each span since the overall line rating is determined by the minimum capacity of line spans. Accordingly, the overall rating of the transmission line is calculated as follows:

$I=\min \left(I_{i}\right)$

where, $I_{i}$ is the ampacity estimated at $i$-th line span.

\subsection{ESS Model}

In this paper, fast-response ESSs are used, since they can successfully follow the fast variations of the load and RESs generation [25]. The dynamics model of fast-response ESSs can be represented as follows:

$$
\begin{aligned}
& S_{i+1}=S_{i}+a_{c} C_{i}-\left(1 / a_{d}\right) D_{i}, \quad i=1,2,3 \ldots \\
& 0 \leq S_{i} \leq S_{\max } \\
& 0 \leq C_{i} \leq C_{\max } \\
& 0 \leq D_{i} \leq D_{\max } \\
& S_{i}=0 \\
& \alpha_{c}, \alpha_{d} \in(0,1)
\end{aligned}
$$

where, $S_{\max }, C_{\max }$, and $D_{\max }$ are the maximum storable energy by the ESS (in MWh), the maximum input (charging) power (in $\mathrm{MW}$ ) and the maximum output (discharging) power (in $\mathrm{MW})$, respectively. The charging efficiency $\left(\alpha_{c}\right)$ is the ratio of the charged power to the input power. Also, the discharging efficiency $\left(\alpha_{d}\right)$ is the ratio of the output power to the discharged power.

\subsection{Wind Farm Model}

The output power of a wind turbine can be determined by using its power curve [26], as shown in Fig. 1. The mathematical expression of the power curve is presented by (10). 


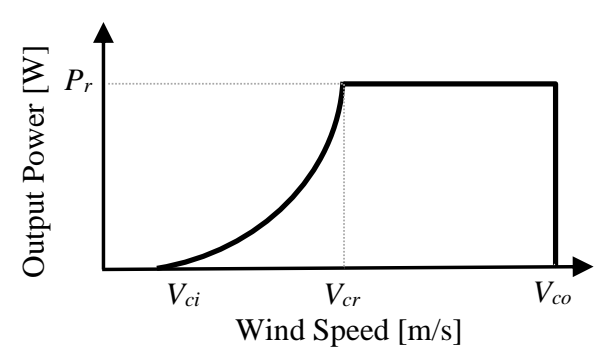

Fig. 1. Power curve of a wind turbine generator

$$
P\left(V_{W}\right)=\left\{\begin{array}{lc}
0 & 0 \leq V_{W} \leq V_{c i} \\
\left(A+B V_{W}+C V_{W}^{2}\right) P_{r} & V_{c i} \leq V_{W} \leq V_{r} \\
P_{r} & V_{r} \leq V_{W} \leq V_{c o} \\
0 & V_{W} \geq V_{c o}
\end{array}\right.
$$

where, $V_{w}$ and $P_{r}$ and are wind speed and rated power of wind turbine, respectively. In addition, $V_{c i}, V_{r}$, and $V_{c o}$ are cut-in, rated and cut-out speed of the turbine, respectively. Moreover, the coefficients $A, B$, and $C$ can be obtained as follows:

$$
\begin{aligned}
& A=\frac{1}{\left(V_{c i}-V_{r}\right)^{2}}\left[V_{c i}\left(V_{c i}+V_{r}\right)-4\left(V_{c i} V_{r}\right) \frac{\left(V_{c i}+V_{r}\right)^{3}}{2 V_{r}}\right] \\
& B=\frac{1}{\left(V_{c i}-V_{r}\right)^{2}}\left[4\left(V_{c i}+V_{r}\right) \frac{\left(V_{c i}+V_{r}\right)^{3}}{2 V_{r}}-\left(3 V_{c i}+V_{r}\right)\right] \\
& C=\frac{1}{\left(V_{c i}-V_{r}\right)^{2}}\left[2-4 \frac{\left(V_{c i}+V_{r}\right)^{3}}{2 V_{r}}\right]
\end{aligned}
$$

\subsection{Solar Power Plant Model}

The output power of a PV generation unit depends on different factors like the solar irradiance and ambient temperature of the site and the parameters of PV modules [27$28]$. The output power of the PV module at the solar irradiance $s$ can be expressed as follows:

$$
\begin{aligned}
& P_{P V o}(s)=N \times F F \times V_{y} \times I_{y} \\
& F F=\frac{V_{M P P} \times I_{M P P}}{V_{o c} \times I_{S C}} \\
& V_{y}=V_{o c}-K_{v} \times T_{c y} \\
& I_{y}=s\left[\mathrm{I}_{S C}+K_{i} \times\left(\mathrm{T}_{c y}-25\right)\right] \\
& T_{c y}=T_{A}+s\left(\frac{N_{O T}-20}{0.8}\right)
\end{aligned}
$$

where, $N$ is the number of PV modules. Additionally, $T_{c y}$ and $T_{A}$ are the cell and ambient temperatures (C), respectively.
Also, $K_{i}$ and $K_{v}$ are the temperature coefficients of the current and voltage $\left(\mathrm{A} /{ }^{\circ} \mathrm{C}\right.$ and $\left.\mathrm{V} /{ }^{\circ} \mathrm{C}\right)$, respectively. In addition, $N_{O T}$ is the nominal operating temperature of cells $\left({ }^{\circ} \mathrm{C}\right)$ and $F F$ is fill factor. Moreover, $V_{O C}$ and $V_{S C}$ are open-circuit voltage and short-circuit current, respectively. As well as, $V_{M P P}$ and $I_{M P P}$ are the voltage and current of the maximum power point.

\subsection{Security Constrained Unit Commitment (SCUC) with DC Load Flow and DTR}

Optimal Power Flow (OPF) is a well-known challenging optimization problem which is non-convex by nature. In this regard, several approaches, like Semi-Definite Programming (SDP), Second-order Cone Programming (SOCP) relaxations, and linearization methodology using the Newton-Raphson methodology, have been presented. Also, a linearized DC load flow has been introduced in [29]. This linearized DC model assumes that:

i. The line susceptance is large compared to the conductance, as described in (19).

ii. The phase angle differences are small enough to be approximated by (20) and (21).

iii. The voltage magnitudes are close to 1.0 as given in (22) and do not vary significantly.

$|g|<<|b|$

$\cos \left(\Theta_{n}^{o}-\Theta_{m}^{o}\right) \gg 1.0$

$\sin \left(\Theta_{n}^{0}-\Theta_{m}^{0}\right) \approx \Theta_{n}^{0}-\Theta_{m}^{0}$

$|\tilde{V}| \approx 1$

By using the above-mentioned approximations, the active power flow of the transmissions lines can be written as expressed in (23) and reactive power flow would be equal to zero. It should be noted that the mentioned approximation is considered to be valid for ESSs, since they affect active power, and their impact on reactive power is negligible [31].

$$
P_{n m}=-b_{n m}\left(\Theta_{n}^{0}-\Theta_{m}^{0}\right)
$$

In this paper, the methodology of [24] is adapted to the SCUC problem with DTR and named DC-SCUC problem. As given by (24), the DC-SCUC problem cost function includes the total production cost of the generation units and their respective reserve provision costs, their start-up and shutdown costs and VOLL of the load shedding. The mixedinteger linear limits for each unit are presented in (25)-(26). The maximum power output of the unit $g$ is also constrained by ramp-up and startup ramp rates as given in (27), as well as by shutdown ramp rates presented in (28). Furthermore, rampdown limits, imposed on the power output, are given in (29). The Minimum up/down-time constraints are described in (30)-(37) according to [31]. The active power balance 
equations that include unit's generations, wind, and solar plant productions, charging and discharging of ESSs, load demand and load shedding variable, are expressed in (38) and (39). The maximum allowable active power flow of transmission lines in STR and DTR states is given by (40)-(41). In (42)(43), the generation limits of wind and solar plants are described. Additionally. ESS constraints are presented in (45).

$$
\begin{aligned}
\min \sum_{t} \sum_{g} & C_{g}^{p}\left(P_{g, t}^{G}, u_{g, t}^{G}\right)+C_{g}^{R}\left(P_{g, t}^{u p}, P_{g, t}^{d n}\right) \\
& +C_{g}^{s u}\left(u_{g, t}^{G}\right)+C_{g}^{s d}\left(u_{g, t}^{G}\right)+\operatorname{VOLL}\left(l s_{t}\right)
\end{aligned}
$$

Subjects to:

$$
\begin{aligned}
& P_{g, \text { min }}^{G} U_{g, t}^{G} \leq P_{g, t}^{G} \leq p_{g, t}^{\max }, \quad \forall \mathrm{g} \in \mathrm{G}, \forall \mathrm{t} \in \mathrm{T} \\
& 0 \leq p_{g, t}^{\max } \leq P_{g, \max }^{G} U_{g, t}^{G} \quad, \quad \forall g \epsilon G, \forall t \in T \\
& p_{g, t}^{\max } \leq P_{g, t-1}^{G}+R U_{g}\left(U_{g, t-1}^{G}\right)+ \\
& S U_{g}\left(U_{g, t}^{G}-U_{g, t-1}^{G}\right)+P_{g, \max }^{G}\left(1-U_{g, t}^{G}\right) \\
& p_{g, t}^{\max } \leq P_{g, \text { max }}^{G} U_{g, t+1}^{G}+S D_{g}\left(U_{g, t}^{G}-U_{g, t+1}^{G}\right), \\
& \forall g \in G, \forall t=1 \ldots T-1 \\
& P_{g, t-1}^{G}-P_{g, t}^{G} \leq R D_{g} U_{g, t}^{G}+S D_{g}\left(U_{g, t-1}^{G}-U_{g, t}^{G}\right) \\
& +P_{g, \max }^{G}\left(1-U_{g, t-1}^{G}\right), \forall g \epsilon G, \forall t \in K \\
& \sum_{t=1}^{G_{s}}\left(1-U_{g, t}^{G}\right)=0, \forall g \in G \\
& \sum_{n=t}^{t+U T_{g}-1} U_{g, n}^{G} \geq U T_{g}\left(U_{g, t}^{G}-U_{g, t-1}^{G}\right), \\
& \forall g \epsilon G, \forall t=G_{g}+1 \ldots T-U T_{g}+1 \\
& \sum_{n=t}^{T}\left[U_{g, n}^{G}-\left(U_{g, t}^{G}-U_{g, t-1}^{G}\right)\right] \geq 0, \forall g \in G, \\
& \forall t=T-U T_{g}+2 \ldots T
\end{aligned}
$$

$\sum_{t=1}^{L_{g}} U_{g, t}^{G}=0, \quad \forall g \in G$

$$
\begin{aligned}
& \sum_{n=t}^{t+D T_{g}-1}\left(1-U_{g, n}^{G}\right) \geq D T_{g}\left(U_{g, t-1}^{G}-U_{g, t}^{G}\right), \\
& \forall g \epsilon G, \forall t=L_{g}+1 \ldots T-D T_{g}+1
\end{aligned}
$$$$
\sum_{n=t}^{T}\left[1-U_{g, n}^{G}-\left(U_{g, t-1}^{G}-U_{g, t}^{G}\right)\right] \geq 0,
$$$$
\forall g \in G, \forall t=T-D T_{g}+2 \ldots T
$$

$$
G_{g}=\min \left[T,\left(\mathrm{UT}_{g}-\mathrm{U}_{g, 0}\right) \mathrm{U}_{g, 0}^{G}\right]
$$

$$
\begin{aligned}
& L_{g}=\min \left[T,\left(D \mathrm{~T}_{g}-S_{g, 0}\right)\left(1-\mathrm{U}_{g, 0}^{G}\right)\right] \\
& \sum_{t}^{T} P_{g, t}^{G}+P_{g, t}^{u p}-P_{g, t}^{d n}+\sum_{t}^{T} p_{P V, t}+\sum_{t}^{T} p_{w, t} \\
& +\sum_{t}^{T} \beta_{t}-\gamma_{t}=\sum_{t}^{T} P_{t}^{D}+l s_{t} \\
& P_{\mathrm{jk}, \mathrm{t}}^{l}=B_{j k, t} \theta_{j k, t} \\
& -P_{j k, \max }^{S T R_{l}} \leq P_{j k, t}^{l} \leq P_{j k, \max }^{S T R_{l}} \\
& -P_{j k, \max }^{D T R_{l}} \leq P_{j k, t}^{l} \leq P_{j k, \max }^{D T R_{l}} \\
& p_{P V, t} \leq P_{P V, t}^{M a x} \\
& p_{w, t} \leq P_{w, t}^{M a x} \\
& U_{g, t}^{G}= \begin{cases}1 & \text { if unit } g \text { is on } \\
0 & \text { if unit } g \text { is off }\end{cases}
\end{aligned}
$$

And (4)-(9)

Since the DC-SCUC problem is a Mixed-Integer Quadratically Constrained programming (MIQCP) problem, it can be easily solved using optimization software. In our case, it is solved by using the Mosek package [31] via the MATLAB interface YALMIP [32].

\subsection{Optimal Allocation of RES and ESS}

The Matpower test systems are designed to have high transmission capacity, which is not suitable for investigating the problems caused by the line congestion [33]. Thus, in this paper, the system loads are increased up to 1.5 times of the base values and also all the transmission lines ratings are reduced to half of the original values. Here, two RESs (i.e. one solar park and one wind farm) are considered to be added to the test systems for supplying the increased system loads and minimizing the active power generation cost. Note that the total capacities of $35 \%$ and $26 \%$ of the maximum load demand are considered for RESs in IEEE 30- and 24-bus systems, respectively. These RESs are optimally allocated by the Genetic algorithm (GA) presented in [34]. Also, optimal placement and sizing of the ESS devices are performed by this algorithm. Generally, the metaheuristic algorithms such as GA detect the optimum points in optimization problems by generating random numbers [35]. These algorithms start with randomly generated solution samples and then improve the solutions.

Here, the objective function for optimal placement and sizing of RESs and ESSs is presented in (24). The investment costs of ESS and DTR devices are given in Table 1 [36]. The GA parameters are given in Table 2. Note that the optimal allocation of RESs and ESSs is performed without considering any DTR on the lines. 
Table 1. Investment cost of ESS technology

\begin{tabular}{|c|c|}
\hline Technology/Device & Cost $\mathbf{( \$ K )}$ \\
\hline ESS $(500 \mathrm{kWh}, 500 \mathrm{~kW})$ & $233.55 /$ year/unit \\
\hline DTR & $31.14 /$ year/line \\
\hline
\end{tabular}

Table 2. Genetic Algorithm parameters

\begin{tabular}{|c|c|}
\hline Parameters & Value \\
\hline Population size-number & 10 \\
\hline Number of genes & Bus size \\
\hline Cross over-rate & 0.7 \\
\hline Mutation rate at Start & 0.12 \\
\hline Mutation rate at End & 0.08 \\
\hline Replacement rate in Elitism Strategy & 0.1 \\
\hline
\end{tabular}

\subsection{Proposed method of DTR technology line selection}

According to [23], the candidate lines for implementing DTR can be selected based on overload and fuel costs. However, a new DTR line selection method is developed here, which considers the effect of each line on load shedding and system costs, including fuel cost and curtailment of RESs. The proposed method consists of two steps as illustrated in Fig. 2.

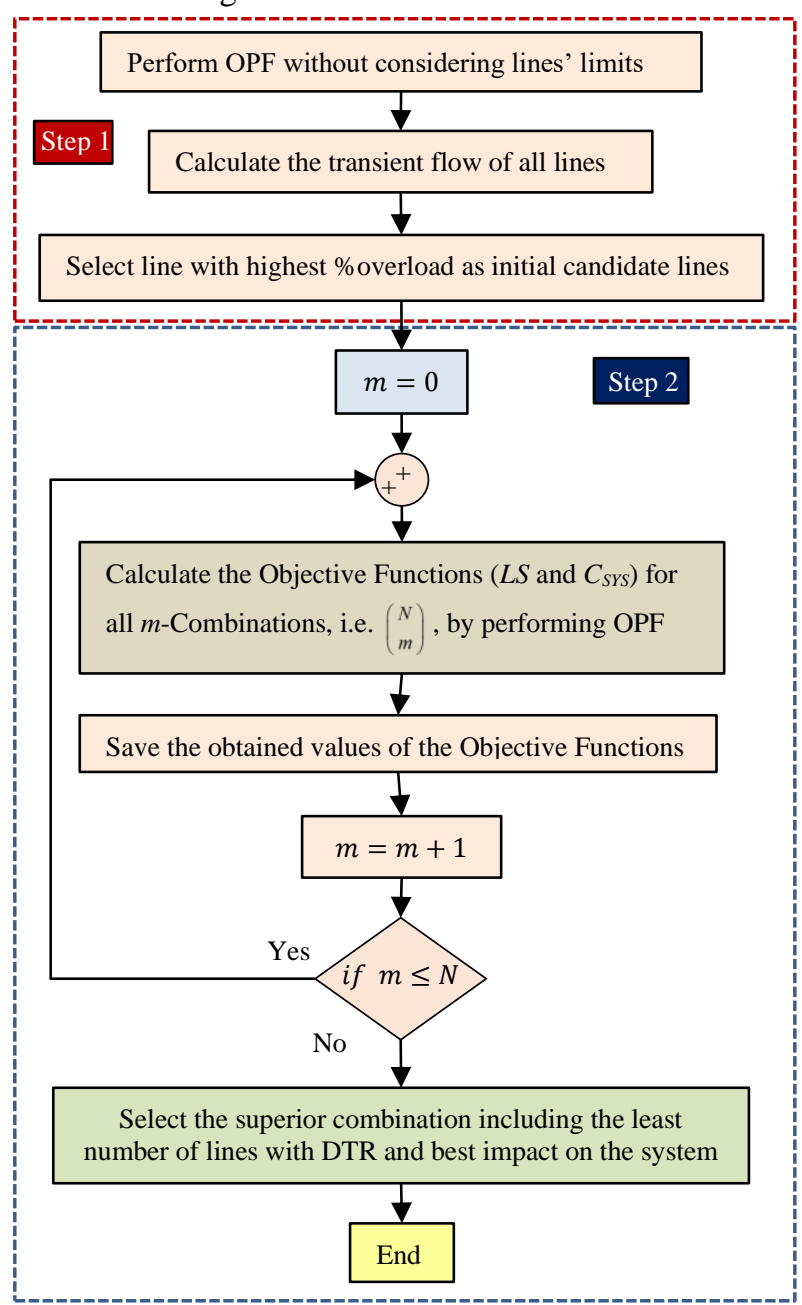

Fig. 2. Flowchart of the proposed DTR Line selection method for DTR technology
In Step 1, optimal power flow is performed without considering the limits of the lines and the transient flows of all lines are calculated. Then, lines with overload are selected as the initial candidate lines. In step 2, the Load Shedding $(L S)$ and System Cost $\left(C_{S Y S}\right)$ are calculated by implementing DTR technology on one, two and all candidate lines selected in step 1. The best combination of the selected lines in step 1, with considerable impact on minimizing the $L S$ and $C_{S Y S}$, is selected as the final candidate lines. In Fig. 2, the $m$ combination refers to select $m$ lines from the initial candidate lines without repetition. In addition, $N$ presents the number of the initial candidate lines.

\subsection{Evaluation Criteria}

Considering the RESs integration optimization, minimization of network costs (generation and load shedding costs) minimization and load shedding minimization, as the main aims of this paper, three evaluation criteria can be expressed as below:

1. Amount of curtailed energy in the wind farm and solar park:

$$
\begin{aligned}
& \text { Curtailment }^{\text {wind }}=\sum_{h=1}^{8760} W P_{h} \quad(M W h / y r) \\
& \text { (46) } \\
& \text { Curtailment }^{\text {solar }}=\sum_{h=1}^{8760} P V P_{h} \quad(M W h / y r)
\end{aligned}
$$

where, $W P_{h}$ and $P V P_{h}$ are the curtailments of wind and solar powers per hour, respectively.

2. The total load shedding:

This criterion is defined as expressed at the following.

$$
L S^{\text {total }}=\sum_{h=1}^{8760} l s_{h} \quad(M W h / y r)
$$

where, $l s_{h}$ is the load shedding for $h$-th hour. Note that to avoid unnecessary load outages, the load shedding has been added with a large coefficient to (24).

3. The total cost of the networks:

$$
\operatorname{Cos} t^{\text {total }}=\sum_{h=l}^{8760} \operatorname{ECOST}_{h} \quad(\$ / y r)
$$

where, $E C O S T_{h}$ is the system costs for $h$-th hour.

\section{Test Systems}

To study the performance of the proposed method (simultaneous application of DTR technology and ESSs) along with the proposed line selection method IEEE RTS 24bus and IEEE 30-bus system are selected as presented in Figs. 3 and 4 . These systems data including generators, buses, and lines, are given in [37]. The climate data used in this paper are the weather data of Tabriz, Iran, available in [38]. Since the selected day is in the spring, the temperature of the STR mode 
is considered to be equal to the maximum temperature of that season so radiation, ambient temperature, and wind speed are $900\left(\mathrm{w} / \mathrm{m}^{2}\right), 20\left({ }^{\circ} \mathrm{C}\right)$, and $0.6(\mathrm{~m} / \mathrm{s})$, respectively. Additionally, the hourly profiles of the ambient temperature-load demand and wind speed-solar irradiation used for these case studies, are shown in Figs. 5 and 6, respectively.

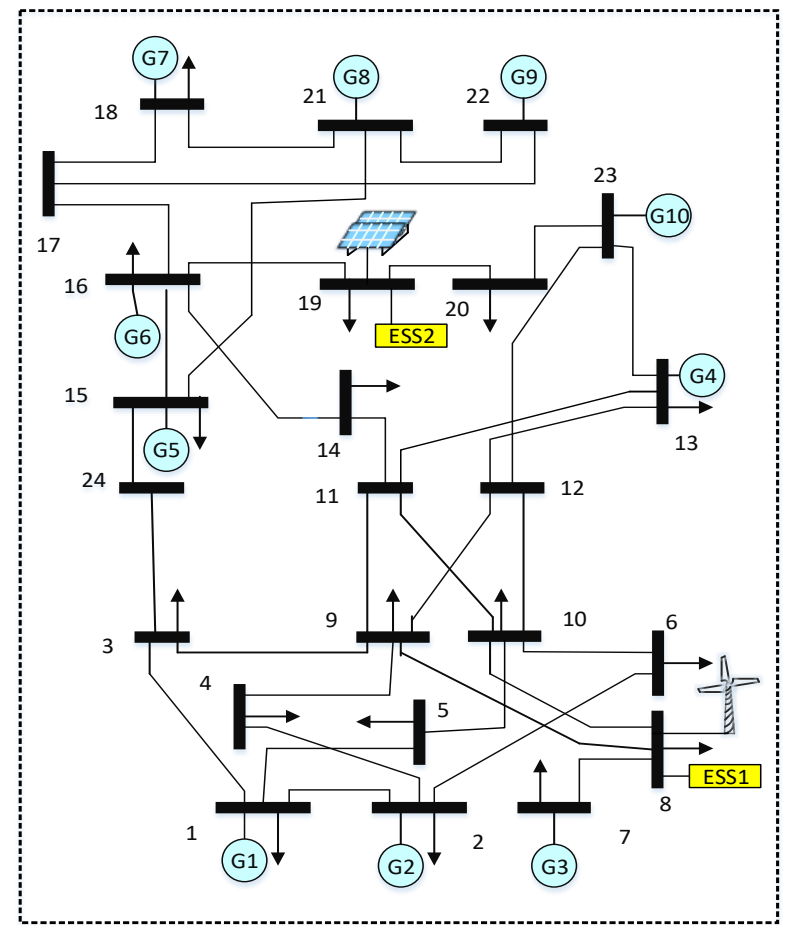

Fig. 3. Single-line diagram of IEEE RTS 24-bus

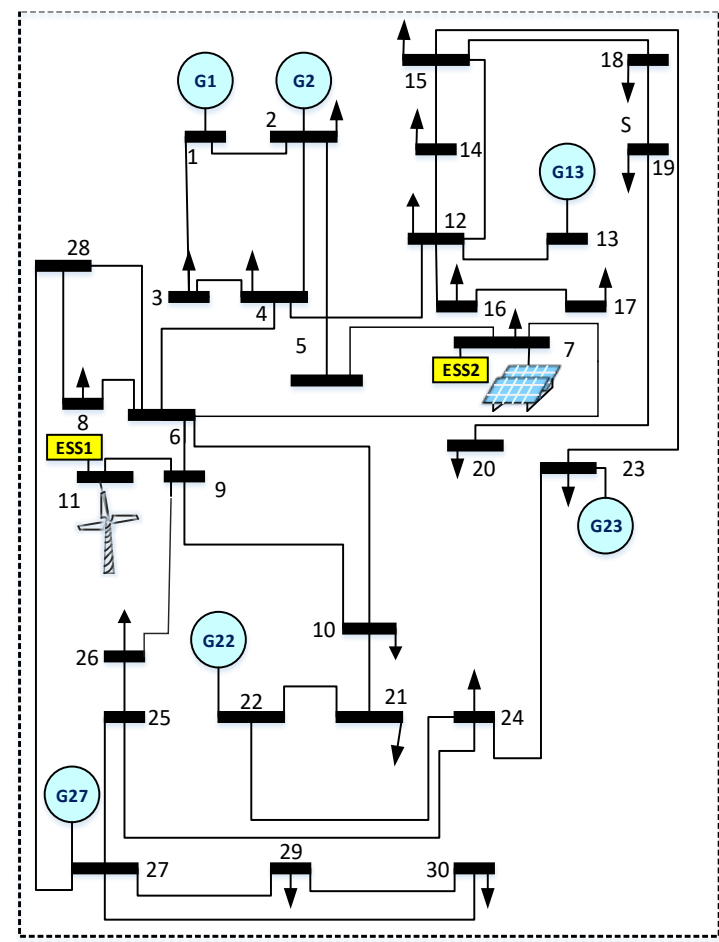

Fig. 4. Single-line diagram of IEEE 30-bus
The maximum MVA rating of conductors is calculated of $75\left[{ }^{\circ} \mathrm{C}\right]$ as the maximum conductor temperatures, and the selected transmission lines are rated hourly based on the weather parameters of their location.

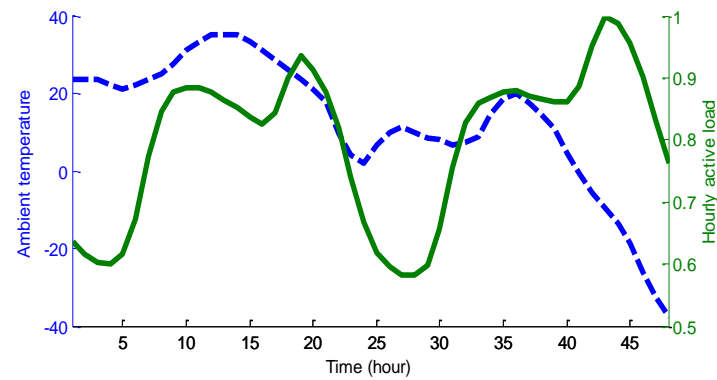

Fig. 5. Active load ambient temperature profiles

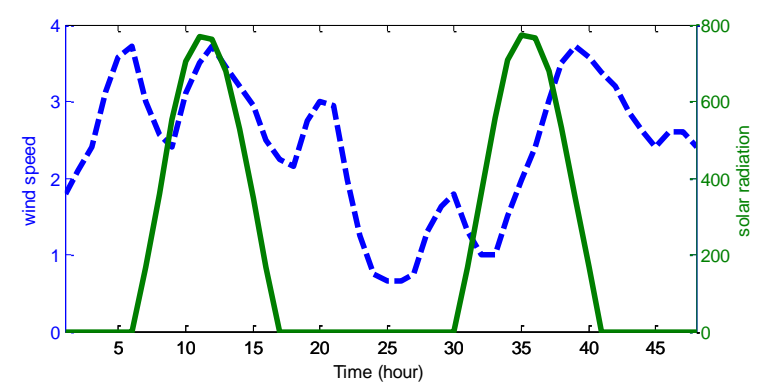

Fig. 6. Wind speed and solar radiation profiles

\section{Simulation Results}

In this section, simulation results of the proposed method along with the developed line selection methodology are presented on the aforementioned test systems. In subsection 4.1, optimal allocation results of RESs and ESSs are presented. Then, the DTR line selection results are presented in sub-section 4.2. After that, in sub-section 4.3, the results of the proposed method (i.e. simultaneous application of DTR and ESS) are presented and compared in four different scenarios.

\subsection{Optimal Allocation of RESs and ESSs}

As mentioned, RESs and ESSs are sized and placed to increase the penetration of renewable energies and decrease load shedding. Here, the RESs optimal placement is performed at the first step and then, ESS devices are optimally allocated. Tables 3 and 4 lists the optimal allocation results of ESS devices and RESs obtained by GA according to the explanations given in Section 2.6.

Table 3. ESSs and RESs data

\begin{tabular}{|c|c|c|c|c|}
\hline \multirow{2}{*}{$\begin{array}{c}\text { Case } \\
\text { study }\end{array}$} & \multicolumn{2}{|c|}{ RESs } & \multicolumn{2}{c|}{ ESSs } \\
\cline { 2 - 5 } & $\begin{array}{c}\text { PV } \\
{[\mathbf{M W}]}\end{array}$ & $\begin{array}{c}\text { Wind } \\
{[\mathbf{M W}]}\end{array}$ & $\begin{array}{c}\text { Rated energy } \\
{[\mathbf{M W h}]}\end{array}$ & $\begin{array}{c}\text { Rated power } \\
{[\mathbf{M W}]}\end{array}$ \\
\hline 24 bus & 500 & 450 & 2000 & 100 \\
\hline 30 bus & 75 & 60 & 500 & 30 \\
\hline
\end{tabular}

Table 4. Results of optimal placement

\begin{tabular}{|c|c|c|c|c|c|c|}
\hline $\begin{array}{c}\text { Case } \\
\text { study }\end{array}$ & PV & Wind & $\begin{array}{c}\text { Objective } \\
\text { Function }\end{array}$ & ESS1 & ESS2 & $\begin{array}{c}\text { Objective } \\
\text { Function }\end{array}$ \\
\hline 24 bus & Bus 8 & Bus 19 & 15028.9 & Bus 8 & Bus 19 & 11138.2 \\
\hline 30 bus & Bus 7 & Bus 11 & 56.364 & Bus 7 & Bus 11 & 54.896 \\
\hline
\end{tabular}




\subsection{Candidate Lines Selection for DTR Based on the Proposed Methodology}

In this section, the candidate lines for implementing DTRs are determined based on the method proposed in Section 2.7. According to the flowchart shown in Fig. 2, first, the lines with overload are selected as the initial candidate lines. As shown in Fig. 7, 7 lines (10, 13, 16, 23, 25, 26, and 28) have the \%Overload in the IEEE 24-bus system. In addition, Fig. 8 shows that there are 8 lines (5-8-10-16-22-2932-35) with overload in the IEEE 30-bus system.

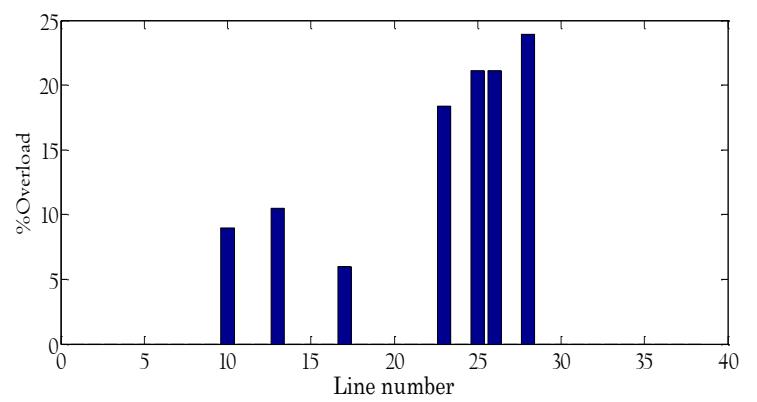

Fig. 7. Overload percent in 24-bus system

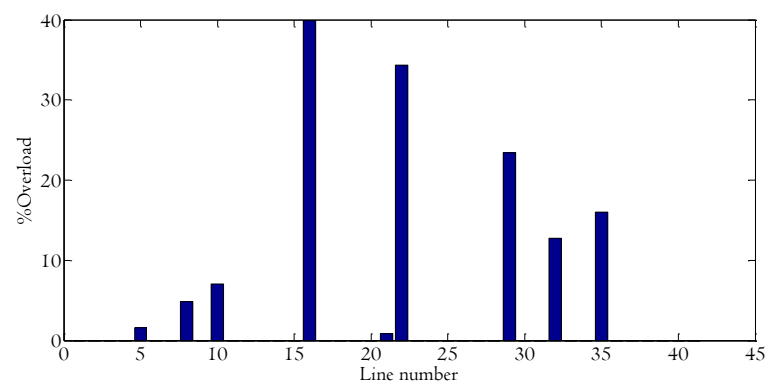

Fig. 8. Overload percent in 30-bus system

As mentioned, these lines should be considered as the initial candidate lines. Now, according to the sec ond step of the proposed method, the DTR should be applied to the initial candidate lines as explained in Section 2.7. The $L S$ and $C_{S Y S}$ values are calculated for the 24-bus system and given in Table 5. As seen, the installation of DTR technology in lines 10, 23, 25,26 and 28 is the best option. The same procedure for the IEEE 30-bus network has also performed and the final lines are obtained as lines $8,10,16,22,29$, and 33. Please note that here, the $L S$ used is calculated when the load is two times the nominal value to determine the lines with the most positive effect on the load shedding minimization.
Table 5. LS and $C_{S Y S}$ calculated for 24-bus system

\begin{tabular}{|c|c|c|c|c|c|c|c|c|}
\hline $\mathbf{L}_{\mathbf{1 0}}$ & $\mathbf{L}_{\mathbf{1 3}}$ & $\mathbf{l}_{\mathbf{1 7}}$ & $\mathbf{L}_{\mathbf{2 3}}$ & $\mathbf{L}_{\mathbf{2 5}}$ & $\mathbf{L}_{\mathbf{2 6}}$ & $\mathbf{L}_{\mathbf{2 8}}$ & $\begin{array}{c}\boldsymbol{L S} \\
(\mathbf{M w})\end{array}$ & $\begin{array}{c}\boldsymbol{C}_{\text {sys }} \\
(\mathbf{\$})\end{array}$ \\
\hline No & No & No & No & No & No & No & 97.98 & $1.88 \times 10^{9}$ \\
\hline Yes & No & No & No & No & No & No & 74.33 & $1.23 \times 10^{9}$ \\
\hline No & No & Yes & No & No & No & No & 91.21 & $1.78 \times 10^{9}$ \\
\hline No & No & No & No & Yes & No & No & 63.74 & $1.16 \times 10^{9}$ \\
\hline No & Yes & No & No & No & No & Yes & 59.25 & $1.13 \times 10^{9}$ \\
\hline Yes & No & No & No & Yes & Yes & No & 43.48 & $1.01 \times 10^{9}$ \\
\hline No & Yes & No & No & No & Yes & Yes & 51.32 & $1.08 \times 10^{9}$ \\
\hline No & No & Yes & Yes & No & Yes & Yes & 32.78 & $0.95 \times 10^{9}$ \\
\hline Yes & No & No & Yes & Yes & Yes & Yes & 0.321 & $0.22 \times 10^{9}$ \\
\hline Yes & Yes & Yes & Yes & Yes & Yes & Yes & 0.319 & $0.22 \times 10^{9}$ \\
\hline
\end{tabular}

\subsection{Studied Scenarios}

In this section, four different scenarios including the proposed method are studied and their results are comprehensively compared. These scenarios are:

1. STR: lines are rated by STR and there is no ESS in power network (base case).

2. STR+ESS: lines are rated by STR and the power system includes ESSs.

3. DTR: lines are rated by DTR but there is no ESS in systems.

4. DTR+ESS (proposed method): Here, it is proposed to use DTR technology and ESSs simultaneously.

All simulations are carried out for a one-year period. In addition, daily simulations are performed for each scenario and the results are discussed as well.

\subsubsection{Result of IEEE RTS 24-bus}

The daily and annual simulations results of all four scenarios for IEEE 24-bus system are listed in Table 6. As seen, the highest amounts of operational costs, load shedding, and energy curtailments belong to Scenario 1 (STR) and the least ones belong to Scenario 4 (DTR+ESS). In other words, the simultaneous use of ESSs and DTR technology improves network performance by increasing the penetration of renewable resources and decreasing the load shedding.

In the following, the injected power of each renewable unit into the system, daily demand, and the energy stored in ESSs for all the scenarios are presented and compared. The comparison of the injected power and generated power for wind unit in different scenarios are shown for two days in Fig 9. It is clear that the amount of curtailment in Scenario 4 (DTR + ESS) is less than the others. In other words, the simultaneous

Table 6. Simulation results for IEEE RTS 24-bus

\begin{tabular}{|c|c|c|c|c|c|c|c|c|}
\hline \multirow{2}{*}{$\begin{array}{l}\text { Case } \\
\text { 24-bus }\end{array}$} & \multicolumn{4}{|c|}{ Daily results } & \multicolumn{4}{|c|}{ Annual results } \\
\hline & $\begin{array}{c}\text { Cost }^{\text {total }} \\
(\$)\end{array}$ & $\begin{array}{l}\text { Curtailment wind } \\
\text { (MWh) }\end{array}$ & $\begin{array}{l}\text { Curtailment } \\
\text { (MWh) }\end{array}$ & $\begin{array}{l}\boldsymbol{L} \boldsymbol{S}^{\text {total }} \\
\text { (MWh) }\end{array}$ & $\begin{array}{c}\text { Cost }{ }^{\text {total }} \\
(\$)\end{array}$ & $\begin{array}{l}\text { Curtailment wind } \\
\text { (MWh) }\end{array}$ & $\begin{array}{l}\text { Curtailment } \\
\text { (MWh) }\end{array}$ & $\begin{array}{l}L S^{\text {total }} \\
\text { (MWh) }\end{array}$ \\
\hline STR & $3.4907 \times 10^{5}$ & $3.7351 \times 10^{3}$ & $1.3945 \times 10^{3}$ & $1.88 \times 10^{3}$ & $1.4907 \times 10^{9}$ & $1.3351 \times 10^{6}$ & $4.945 \times 10^{5}$ & $6.88 \times 10^{5}$ \\
\hline STR+ESS & $1.9765 \times 10^{5}$ & $2.6573 \times 10^{3}$ & $4.183 \times 10^{2}$ & $1.13 \times 10^{3}$ & $6.9765 \times 10^{7}$ & $9.6573 \times 10^{5}$ & $1.483 \times 10^{5}$ & $4.13 \times 10^{5}$ \\
\hline DTR & $5.2757 \times 10^{4}$ & $4.496 \times 10^{2}$ & $7.446 \times 10^{2}$ & $3.12 \times 10^{2}$ & $1.8757 \times 10^{7}$ & $1.496 \times 10^{5}$ & $2.146 \times 10^{5}$ & $1.12 \times 10^{5}$ \\
\hline DTR+ESS & $3.2371 \times 10^{4}$ & $0.179 \times 10^{2}$ & $0.206 \times 10^{2}$ & $0.107 \times 10^{2}$ & $1.1371 \times 10^{7}$ & $7.339 \times 10^{3}$ & $6.206 \times 10^{3}$ & $3.707 \times 10^{3}$ \\
\hline
\end{tabular}


application of DTR and ESS is the best option since it enables system operators to use the maximum capacity of the generation unit. Moreover, the injected and generated power/energy of the solar unit for different scenarios are shown in Fig 10 for two days. Again, the best scenario is the fourth one, since the solar park is maximally utilized in this scenario. Since RESs penetration in Scenario 4 is higher than the others, this scenario will lead to less active power generation costs.

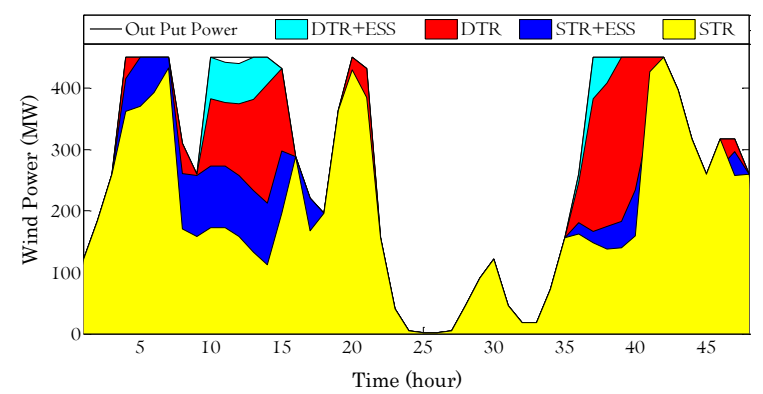

Fig. 9. Wind power injected to system for case 24-bus system

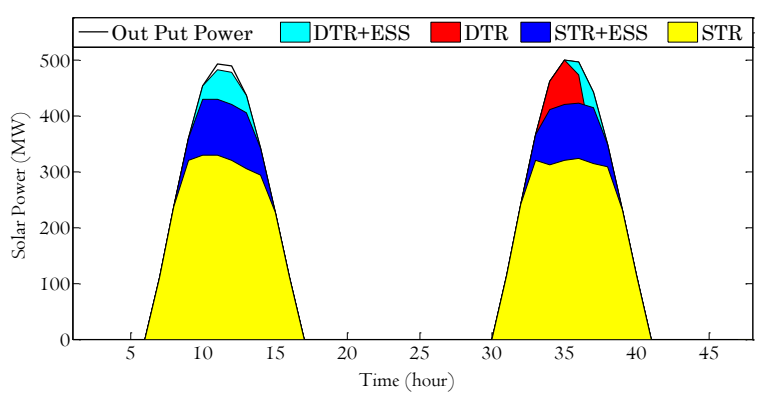

Fig. 10. Solar power injected to system for case 24-bus system

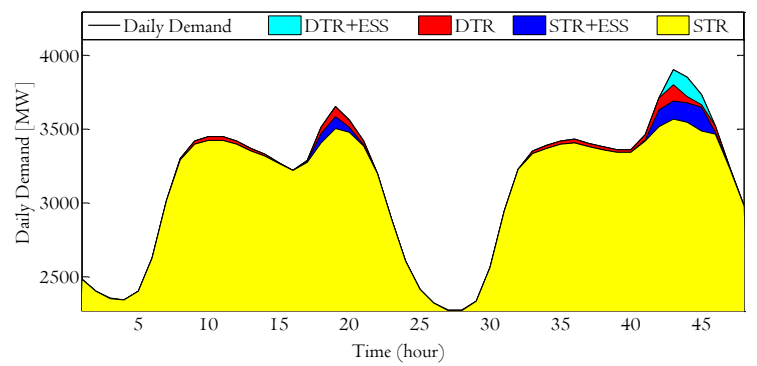

Fig. 11. Load demand profile for case 24-bus system

The amount of the supplied demand for two days is shown in Fig. 11 for different scenarios. As seen, the amount of not supplied demand in Scenario 4 (DTR+ESS) is lower than the other scenarios since it can overcome both problems of active power supply shortage and lines capacity inadequacy at the same time. The lack of power generation at peak periods is solved by ESS devices which store the excessive generation of RESs and inject it in peak periods. Moreover, the lines capacity inadequacy is solved using DTR technology. Fig.12 shows the amount of the stored energy in the ESSs. As shown, the area under the graph (i.e. total stored energy) is greater for Scenario 4 compared to the others. The total costs of the different scenarios are shown in Fig. 13. As seen, the overall system cost of Scenario 4 is lower than that of other scenarios.

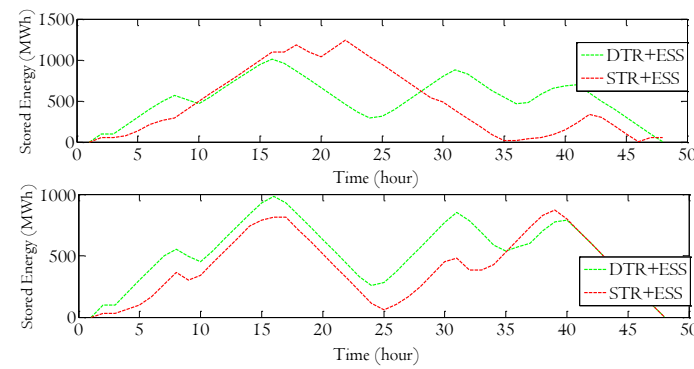

Fig. 12. Stored energy in ESSs for case 24-bus system

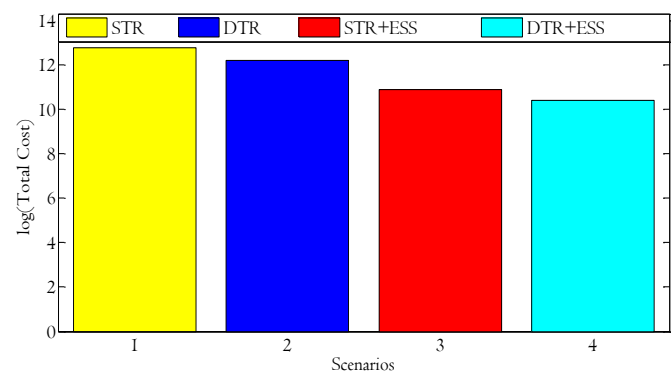

Fig. 13. Total cost for case 24-bus system

\subsubsection{IEEE 30-bus system}

The daily and annual results of the four scenarios on the IEEE 30-bus system are presented in Table 7. Like the presented results in the previous section, the least amount of curtailments and load shedding are obtained in Scenario 4 which means that the proposed method is the best option. The injected power amount by wind and solar units to the system in different scenarios is respectively shown in Figs. 14 and 15 for two consecutive days. As clearly shown in Fig. 14, it is feasible to utilize the maximum output power of wind units only by using ESSs in the first day. However, during the second day, it is required to use DTR technology along with ESSs to be able to utilize the maximum output power of wind units. According to Fig. 15, by using the proposed method, the system operators can optimally use the solar units. The

Table 7. Simulation results for IEEE 30-bus

\begin{tabular}{|c|c|c|c|c|c|c|c|c|}
\hline \multirow{2}{*}{$\begin{array}{l}\text { Case } \\
\text { 30-bus }\end{array}$} & \multicolumn{4}{|c|}{ Daily results } & \multicolumn{4}{|c|}{ Annual results } \\
\hline & $\begin{array}{c}\text { Cost }^{\text {total }} \\
(\$)\end{array}$ & $\begin{array}{l}\text { Curtailment } \\
\text { (MWhd } \\
\text { (MWh) }\end{array}$ & $\begin{array}{l}\text { Curtailment }{ }^{\text {Solar }} \\
\text { (MWh) }\end{array}$ & $\begin{array}{l}\boldsymbol{L} \boldsymbol{S}^{\text {total }} \\
\text { (MWh) }\end{array}$ & $\begin{array}{c}\text { Cost }^{\text {total }} \\
(\$)\end{array}$ & $\begin{array}{l}\text { Curtailment }{ }^{\text {wind }} \\
\text { (MWh) }\end{array}$ & $\begin{array}{l}\text { Curtailment Solar } \\
\text { (MWh) }\end{array}$ & $\begin{array}{l}L S^{\text {total }} \\
(\mathbf{M W h})\end{array}$ \\
\hline STR & $2.0945 \times 10^{5}$ & $4.2530 \times 10^{2}$ & $2.7648 \times 10^{2}$ & $5.23 \times 10^{2}$ & $7.02 \times 10^{7}$ & $1.53 \times 10^{5}$ & $9.80 \times 10^{4}$ & $1.77 \times 10^{5}$ \\
\hline STR+ESS & $1.350 \times 10^{5}$ & $2.6096 \times 10^{2}$ & $0.1765 \times 10^{2}$ & $2.19 \times 10^{2}$ & $4.36 \times 10^{7}$ & $9.22 \times 10^{4}$ & $5.42 \times 10^{4}$ & $7.63 \times 10^{4}$ \\
\hline DTR & $8.0909 \times 10^{3}$ & $2.0995 \times 10^{2}$ & $2.446 \times 10^{2}$ & $0.68 \times 10^{2}$ & $2.84 \times 10^{6}$ & $7.95 \times 10^{4}$ & $8.10 \times 10^{4}$ & $2.54 \times 10^{4}$ \\
\hline DTR+ESS & $8.2963 \times 10^{2}$ & $0.34 \times 10^{2}$ & $2.06 \times 10^{2}$ & $0.059 \times 10^{2}$ & $2.95 \times 10^{5}$ & $1.87 \times 10^{4}$ & $2.2 \times 10^{4}$ & $0.30 \times 10^{4}$ \\
\hline
\end{tabular}




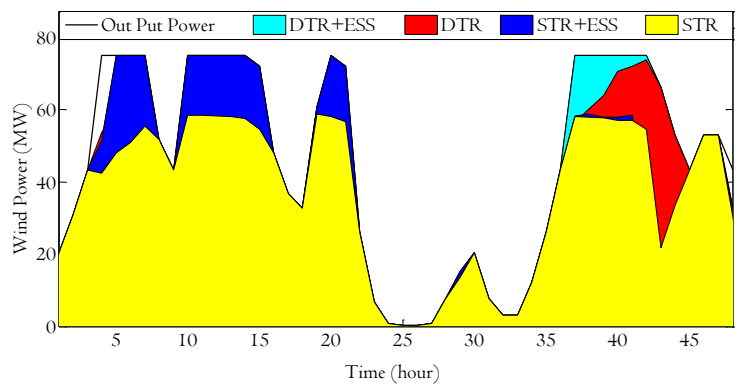

Fig. 14. Wind power injected to system for case 30-bus system

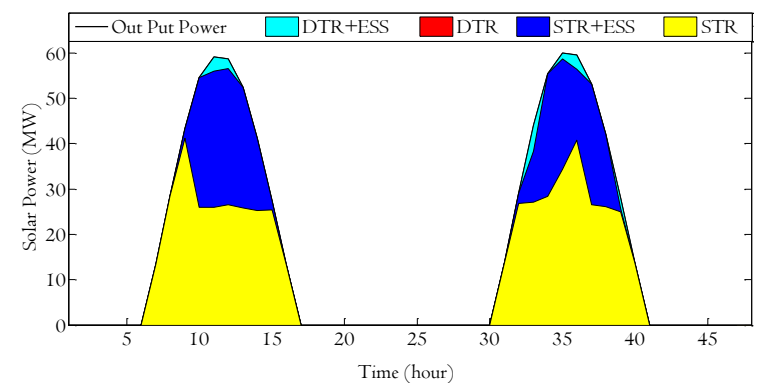

Fig. 15. Solar power injected to system for case 30-bus system

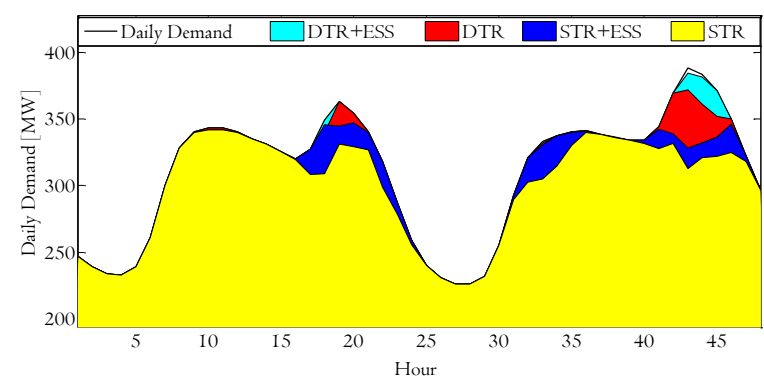

Fig. 16. Load demand profile for case 30-bus system
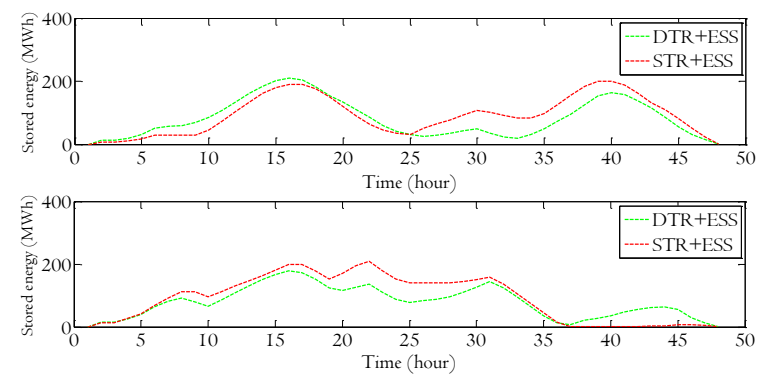

Fig. 17. Stored energy in ESSs for case 30-bus amount of the supplied demand, the amount of the stored energy in the ESSs, and the total costs for different scenarios are respectively shown in Figs. 16-18. It is obvious that the proposed method, i.e. Scenario 4 , is the superior one and the best option.

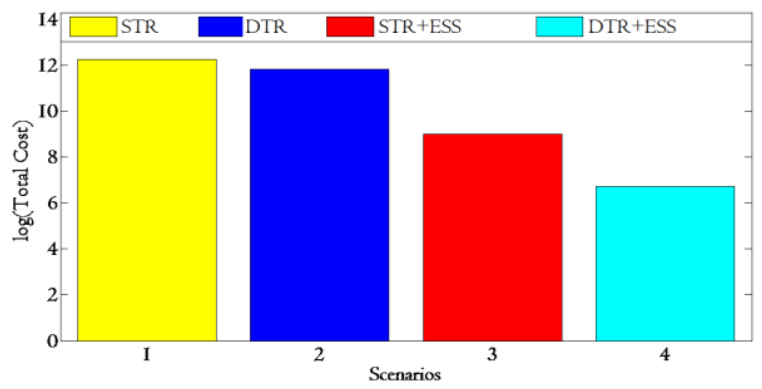

Fig. 18. Total cost for case 30-bus system

As clearly shown in the results, the simultaneous application of ESSs and DTR technology has a more, positive effect on IEEE 30 bus system as the IEEE 24 bus system. In other words, the proposed method is more effective in complex systems.

To gain a better understanding, in Table 8 , the obtained improvements of Scenarios 2, 3 and 4 are compared to Scenario 1 (STR). As clearly seen, among the compared Scenarios, the proposed solution, i.e. Scenario 4 (ESS+DTR), is absolutely the superior one. In IEEE 24 and 30 bus systems, the proposed method successfully decreases the total generation cost about $90.72 \%$ and $94.61 \%$, respectively. Besides, the RESs' integration is augmented up to $96.52 \%$ compared to Scenario 1. Also, the load shedding is reduced by about $98.87 \%$ and $96.13 \%$ in IEEE 30 and 24 bus systems, respectively. In the following, a brief discussion is given based on the obtained results to evaluate the effectiveness of each technology on the objectives of this study. According to Table 9, in all the comparison categories, the proposed solution is the most efficient method to achieve the defined objetives for the system, i.e. load shedding minimization and optimizing RESs' integration into the system. Based on Table 9, implementation DTR on the transmission lines of the system , i.e. Scenario 3, has the second place in the most effective solutions for all comparison terms, expect for curtailment of solar parks. In the case of reduction in solar curtailment, Scenario 2 (ESS+STR) shows a better performance than Scenario 3 and takes the second place. This

Table 8. Improvement percentage of each scenario compared to Scenario 1 (STR)

\begin{tabular}{|c|c|c|c|c|c|c|}
\hline Test system & scenario & Cost $^{\text {total }}$ & Curtailment $^{\text {wind }}$ & Curtailment $^{\text {solar }}$ & LS $^{\text {total }}$ & Total \\
\hline \multirow{3}{*}{ 24-bus } & ESS & $\% 43.37$ & $\% 28.85$ & $\% 70.01$ & $\% 39.89$ & $\% 45.53$ \\
\cline { 2 - 7 } & DTR & $\% 84.88$ & $\% 87.96$ & $\% 46.60$ & $\% 83.43$ & $\% 75.71$ \\
\cline { 2 - 7 } & DTR+ESS & $\mathbf{\% 9 0 . 7 2}$ & $\% 96.52$ & $\% 94.53$ & $\% 96.13$ & $\% 93.97$ \\
\hline \multirow{3}{*}{ 30-bus } & ESS & $\% 35.56$ & $\% 38.64$ & $\% 83.64$ & $\% 58.64$ & $\% 54.12$ \\
\cline { 2 - 7 } & DTR & $\% 86.13$ & $\% 50.63$ & $\% 11.63$ & $\% 86.63$ & $\% 58.75$ \\
\cline { 2 - 7 } & DTR+ESS & $\mathbf{\% 9 4 . 6 1}$ & $\mathbf{\% 9 1 . 5 1}$ & $\mathbf{\% 9 5 . 8 6}$ & $\mathbf{\% 9 8 . 8 7}$ & $\mathbf{\% 9 7 . 9 6}$ \\
\hline
\end{tabular}


Table 9. The qualitative results of the comparisons

\begin{tabular}{|c|c|c|}
\hline Comparison parameters & Qualitative Effectiveness & Best Solution \\
\hline Cost $^{\text {total }}$ & $($ DTR+ESS $)>$ DTR $>($ ESS+STR $)>$ STR & (DTR+ESS) \\
\hline Curtailment $^{\text {wind }}$ & $($ DTR+ESS $)>$ DTR $>($ ESS+STR $)>$ STR & (DTR+ESS) \\
\hline Curtailment $^{\text {solar }}$ & $($ DTR+ESS $)>($ ESS+STR $)>$ DTR $>$ STR & (DTR+ESS) \\
\hline $\mathbf{L S}^{\text {total }}$ & $($ DTR+ESS $)>$ DTR $>($ ESS+STR $)>$ STR & (DTR+ESS) \\
\hline Total & $($ DTR+ESS $)>$ DTR $>($ ESS+STR $)>$ STR & (DTR+ESS) \\
\hline
\end{tabular}

is due to the fact that the power generation of PV panels depend on the solar radiation. Hence, using ESS devices to save their energy and inject it to the system in the peak hours would be a more effective solution than using onle DTR devices without ESS devices in the system. Comsequently, the proposed solution (using DTR and ESS along with the proposed DTR line selection method) is the most suitable solutions for the modern power systems to increase their RES integration and minimize load shedding.

\section{Conclusion}

In this paper, applications of ESSs along with DTR technology has been proposed to achieve minimum load shedding and generation cost. The main effect on minimizing the total generation cost is performed by the optimal integration of RESs into the system. The location of the ESS installation has been performed by the genetic algorithm, but a new method has been proposed for the allocating of DTR monitoring equipment, which was able to determine the best lines for implementing DTR technology. The proposed method (i.e. using ESSs and DTR technology) has been compared with different scenarios. The results show that ESSs can save curtailed energy due to RESs variable nature and the generator's thermal constraints, and the DTR technology eliminates curtailed energy due to the inadequacy of lines capacity. According to the results, in comparison with STR technology the proposed method can provide $90.72 \%-99.6 \%$ reduction in cost, $93.5 \%-99.52 \%$ reduction in curtailment of wind power, $98.5 \%-99.86 \%$ reduction in curtailment of solar power and, $98.87 \%-99.13 \%$ reduction in load shedding in IEEE 24 and 30-bus test systems, respectively. As seen, it is obvious that the proposed method can improve all the considered objectives was better than other solutions.

\section{References:}

[1] Moriarty, Patrick, and Damon Honnery. "Global renewable energy resources and use in 2050." In Managing Global Warming, pp. 221-235. Academic Press, 2019.

[2] Firouzi, Mehdi, Gevork B. Gharehpetian, and Younes Salami. "Active and reactive power control of wind farm for enhancement transient stability of multi-machine power system using UIPC." IET Renewable Power Generation 11, no. 8 (2017): 1246-1252.
[3] Wiser, Ryan H., and Bolinger, Mark. Thu . "2017 Wind Technologies Market Report". United States. doi: 10.2172/1471044. https://www.osti.gov/servlets/purl/1471044

[4] Bird, Lori, Jaquelin Cochran, and Xi Wang. Wind and solar energy curtailment: Experience and practices in the United States. No. NREL/TP-6A20-60983. National Renewable Energy Lab.(NREL), Golden, CO (United States), 2014.

[5] Heckenbergerová, Jana, and Jirí Hošek. "Dynamic thermal rating of power transmission lines related to wind energy integration." In 2012 11th International Conference on Environment and Electrical Engineering, pp. 798-801. IEEE, 2012.

[6] Wiszniewski, Andrzej. "New criteria of voltage stability margin for the purpose of load shedding." IEEE Transactions on Power Delivery 22, no. 3 (2007): 13671371.

[7] Stephen, Rob, Jean-Louis Lilien, Dale Douglass, Mark Lancaster, Gerhard Biedenbach, George Watt, Ruis Pestana, Patrick Ferrières, and Michael Schmale. Guide for Application of direct real-time monitoring systems. Cigré, 2012.

[8] Johnson, Justin, Cale Smith, Mike Young, Ken Donohoo, Ross Owen, Eddit Clark, Raul Espejo et al. Dynamic line rating oncor electric delivery smart grid program. Oncor Electric Delivery Company Llc, 2013.

[9] Xu, Bolun, Andreas Ulbig, and Göran Andersson. "Impacts of dynamic line rating on power dispatch performance and grid integration of renewable energy sources." In IEEE PES ISGT Europe 2013, pp. 1-5. IEEE, 2013.

[10]Teh, Jiashen, and Ian Cotton. "Reliability impact of dynamic thermal rating system in wind power integrated network." IEEE Transactions on Reliability 65 , no. 2 (2016): 1081-1089.

[11]Fernández Herrero, Elvira, Igor Albizu Flórez, Bedialauneta Landaribar, Miren Terese, Angel Javier Mazón Sainz-Maza, and Patricia T. Leite. "Review of dynamic line rating systems for wind power integration." (2016).

[12] Massaro, Fabio, Mariano Giuseppe Ippolito, Enrico Maria Carlini, and Fabio Bassi. "Maximizing energy transfer and RES integration using dynamic thermal 
rating: Italian TSO experience." Electric Power Systems Research 174 (2019): 105864.

[13] Sun, Wei-Qing, Yan Zhang, Cheng-Min Wang, and Ping Song. "Flexible load shedding strategy considering realtime dynamic thermal line rating." IET Generation, Transmission \& Distribution 7, no. 2 (2013): 130-137.

[14]Dong, Xiaoming, Ruiqi Zhang, Mengxia Wang, Jinyu Wang, Chengfu Wang, Yong Wang, and Peng Wang. "Capacity assessment for wind power integration considering transmission line electro-thermal inertia." International Journal of Electrical Power \& Energy Systems 118 (2020): 105724.

[15]Fernandez, E., I. Albizu, M. T. Bedialauneta, A. J. Mazon, and P. T. Leite. "Dynamic line rating systems for wind power integration." In IEEE Power and Energy Society Conference and Exposition in Africa: Intelligent Grid Integration of Renewable Energy Resources (PowerAfrica), pp. 1-7. IEEE, 2012.

[16] Díaz-González, Francisco, Andreas Sumper, Oriol Gomis-Bellmunt, and Roberto Villafáfila-Robles. "A review of energy storage technologies for wind power applications." Renewable and sustainable energy reviews 16, no. 4 (2012): 2154-2171.

[17]Denholm, P. "Energy storage to reduce renewable energy curtailment." In 2012 IEEE Power and Energy Society General Meeting, pp. 1-4. IEEE, 2012.

[18]Denholm, Paul, and Trieu Mai. "Timescales of energy storage needed for reducing renewable energy curtailment." Renewable energy 130 (2019): 388-399.

[19]Bolufawi, Omonayo. "Renewable Energy Integration with Energy Storage Systems and Safety." Special Topics in Renewable Energy Systems (2018): 39.

[20] Madadi, S., B. Mohammadi-Ivatloo, and S. Tohidi. "Decentralized optimal multi-area generation scheduling considering renewable resources mix and dynamic tie line rating." Journal of Cleaner Production 223 (2019): 883-896.

[21]Teh, Jiashen, and Ian Cotton. "Critical span identification model for dynamic thermal rating system placement." IET Generation, Transmission \& Distribution 9, no. 16 (2015): 2644-2652.

[22] Matus, Marcelo, Doris Sáez, Mark Favley, Carlos Suazo-Martínez, José Moya, Guillermo JiménezEstévez, Rodrigo Palma-Behnke, Gabriel Olguín, and Pablo Jorquera. "Identification of critical spans for monitoring systems in dynamic thermal rating." IEEE Transactions on power delivery27, no. 2 (2012): 10021009.

[23] Chu, R. F. "On selecting transmission lines for dynamic thermal line rating system implementation." IEEE transactions on power systems 7, no. 2 (1992): 612-619.

[24]"IEEE 738-2012", Standard for Calculating the Current-Temperature Relationship of Bare Overhead Conductors, Jun. 2012.

[25] Su, Han-I., and Abbas El Gamal. "Modeling and analysis of the role of fast-response energy storage in the smart grid." In 2011 49th Annual Allerton Conference on
Communication, Control, and Computing (Allerton), pp. 719-726. IEEE, 2011.

[26] Karki, Rajesh, Po Hu, and Roy Billinton. "A simplified wind power generation model for reliability evaluation." IEEE transactions on Energy conversion 21, no. 2 (2006): 533-540.

[27]Hung, Duong Quoc, Nadarajah Mithulananthan, and Kwang Y. Lee. "Determining PV penetration for distribution systems with time-varying load models." IEEE Transactions on Power Systems 29, no. 6 (2014): 3048-3057.

[28] Movahedi, Amir, Abolfazl Halvaei Niasar, and G. B. Gharehpetian. "Designing SSSC, TCSC, and STATCOM controllers using AVURPSO, GSA, and GA for transient stability improvement of a multi-machine power system with PV and wind farms." International Journal of Electrical Power \& Energy Systems 106 (2019): 455-466.

[29]Coffrin, Carleton, and Pascal Van Hentenryck. "A linear-programming approximation of $\mathrm{AC}$ power flows." INFORMS Journal on Computing 26, no. 4 (2014): 718-734.

[30]Carrión, Miguel, and José M. Arroyo. "A computationally efficient mixed-integer linear formulation for the thermal unit commitment problem." IEEE Transactions on power systems 21 , no. 3 (2006): 1371-1378.

[31] MOSEK Optimization Toolbox for MATLAB. [online] Available at:https://docs.mosek.com/8.1/toolbox/installinterface.html

[32] Löfberg, Johan. "YALMIP: A toolbox for modeling and optimization in MATLAB." In Proceedings of the CACSD Conference, vol. 3. 2004.

[33] Shaker, Hamid, Hamidreza Zareipour, and Mahmud Fotuhi-Firuzabad. "Reliability modeling of dynamic thermal rating." IEEE Transactions on Power Delivery 28, no. 3 (2013): 1600-1609.

[34] Xu, Daming, et al. "Optimal sizing of standalone hybrid wind/PV power systemsusing genetic algorithms." Electrical and Computer Engineering, 2005. Canadian Conference on. IEEE, 2005.

[35] Abbasi, Maysam, Abbasi, Ehsan \& MohammadiIvatloo, Behnam. "Single and multi-objective optimal power flow using a new differential-based harmony search algorithm." J Ambient Intell Human Comput (2020). https://doi.org/10.1007/s12652-020-02089-6

[36] Giannelos, Spyros, Ioannis Konstantelos, and Goran Strbac. "Option value of dynamic line rating and storage." In 2018 IEEE International Energy Conference (ENERGYCON), pp. 1-6. IEEE, 2018.

[37]Zimmerman, Ray D., Carlos E. Murillo-Sánchez, and Deqiang Gan. "MATPOWER: A MATLAB power system simulation package." Manual, Power Systems Engineering Research Center, Ithaca NY 1 (1997).

[38] http://pvwatts.nrel.gov/ 